\title{
Clinical research in a nutshell: How to embark on research journey?
}

\author{
Asim Elnour ${ }^{1 *}$, Alaa Abdul Aziz' ${ }^{1}$ Abdulla Shehab², Omar Abdulla Shehab ${ }^{2}$, Akshaya Srikanth \\ Bhagavathula $^{3}$, Pinar Erkekoglu ${ }^{4}$, Farah Hamad ${ }^{5}$, Cristina Sanches Giraud ${ }^{6}$, Saif Al Nuaimi ${ }^{7}$, \\ Aaesha Al Suwaidi ${ }^{8}$, Naama M. S. Alkalbani ${ }^{9}$, Abdelrazek M Ali Abd Elrazek ${ }^{10}$, Mohammed \\ Baraka $^{11}$, Sahar Asim ${ }^{12}$, Rauda Abdulla ${ }^{13}$, Abdulla Al Amoodi ${ }^{13}$ \\ ${ }^{1}$ Department of Pharmacology, College of Medicine and Health Sciences, UAE University, Dubai, UAE \\ ${ }^{2}$ Department of Internal Medicine, College of Medicine and Health Sciences, UAE University, Dubai, UAE \\ ${ }^{3}$ Department of Clinical Pharmacy, University of Gondar College of Medicine and Health Sciences, Gondar, Ethiopia \\ ${ }^{4}$ Department of Toxicology, Faculty of Pharmacy, Hacettepe University, Ankara, Turkey \\ ${ }^{5}$ College of Pharmacy, Ajman University of Sciences and Technology, Ajman-UAE \\ ${ }^{6}$ Federal University of São João del Rei, Campus Midwest Dona Lindu, Divinópolis, Brazil \\ ${ }^{7}$ Department of Internal Medicine, Tawam Hospital, Abu Dhabi Health Services, Seha, UAE \\ ${ }^{8}$ Consultat Endocrinologist, Al Ain Hospital, Al Ain-UAE \\ ${ }^{9}$ Senior Pharmacist, Tawam Hospital, Abu Dhabi Health Services, Seha, UAE \\ ${ }^{10}$ Medicine of Liver Transplantation, and Consultant, GIT and Hepatology, Al-Azhar University, Asiut, Egypt \\ ${ }^{11}$ Clinical pharmacist, Dammam University, Dammam-KSA \\ ${ }^{12}$ College of Dentistry, Ajman University of Sciences and Technology, UAE \\ ${ }^{13}$ College of Medicine and Health Sciences, UAE University, UAE
}

Received: October 1,2015; Accepted: November 13, 2015, Published: December 18, 2015

*Corresponding author: Asim Ahmed Elnour, Associate Professor, Department of Pharmacology, College of Medicine and Health Sciences, UAE University, Dubai, UAE, Tel: 00971506734096; Email: Assahura1962@uaeu.ac.ae

\begin{abstract}
Background: Medical students, interns and residents at all levels needs a boost dose of simple plan and exemplified written format to participate in medical (clinical/toxicological) research.

Aim: Herein, we aim to provide "critical points to take into account when developing a research from the idea to the final publication". This will guide those who need to delve deeper the clinical research panorama and write a clinical research paper.

Methods: We have performed a review on available tools that provide information on how to conduct a research study and develop a manuscript intended for publication. In order to initiate any clinical research activity, an initial assessment of the current routine clinical practice is a pre-requisite to conceive and design the research. In "Introduction", a clinical researcher should inform the readers about the clinical implications of the study and should anticipate new practices. The author should also bring the statistical outputs in organized predefined titled tables, no matter how many initially; however, later may deduce to the minimum number of tables.

Results: The researcher or investigator should provide anticipated results for the proposed protocol and detailed results for the manuscript. The initial results are the demographics and past medical/medication/ morbidities. Then should narrow down more specific to the main findings. Each results illustrated in tables should stands as the discussion area. Therefore, tables must follow an uphill fashion and upward direction to the final conclusions.
\end{abstract}

Discussions: The tip of the iceberg is the main findings and consequently, the successive less important findings should be reported. The discussion is the study background where one can explore the findings and compare the results to the international studies. The aligned differences between the study findings and other studies should be reasoned and explained with clear statements.

Conclusions: The researcher should conclude the most important findings from the work performed and should recommend changes and interventions that facilitate the clinical research findings. The researcher should then state applicability of the findings to day-today clinical practice and generalization to the population from which the data are pertaining.

Keywords: Clinical Research; Structured abstract; Outcome Measure; Cone format Introduction; Medical student

\section{Introduction}

\section{Rational}

Medical students, interns, residents and practitioners at all levels need a boost dose of simple plan and exemplified written format to participate in medical clinical research studies or write a manuscript. There are great opportunities for medical clinical researchers to establish research career in line with their current clinical practice and respective clinical settings early in their stepladder. In order to initiate any clinical research activity, an initial assessment of the current routine clinical practice is a pre-requisite to conceive and design the research plans. Once a specific clinical practice is critically appraised for 
possible interventions, then a research question is emulated. This question resembles the hypothesis and the rationale of the study. The initial step is a hypothesis, rationale or research question in order to investigate, examine, explore, and intervene with the previous, current and/or future clinical practice for possible improvement/change or implementation of new clinical services. The most important questions in introduction is to ask why was the study undertaken? What is the research question, the tested hypothesis or the purpose of the research?

Objectives: This review aimed to provide a guide for writing a clinical research paper to researchers who need to delve deeper in the clinical research panorama. We objectively alleviate the pitfalls involved in abstract writing and offer a proposal where the subheadings given under each of the abstract sections can be further expanded to emerge the manuscript in a stepwise fashion.

Aim: Herein, we aim to provide "critical points to take into account when developing a research from the idea to the final publication". This will guide those who need to delve deeper the clinical research panorama and ultimately write a clinical research paper.

\section{Tailoring the research plan: The research question}

The research proposal/plan/prospectus is the initial step in producing thesis/dissertation/major research project. Intent is to convince a supervisor or academic committee that topic and approach are sound, to obtain approval, proceed and often gain funding. It indicate forming a plan of action, proposal should show theoretical positioning and relationship to past work in research area.

The research question follows to address the gap in knowledge whereby a topic is chosen. It is imperative to do research search on the chosen topic. Discussion of other studies findings, critical appraisal, compare and assess results and justifies your project/ protocol/study/manuscript and finally paper-article-publication.

This will broaden the investigator knowledge about the selected research topic and provide an in depth information about the research from different perspectives. The conceptual framework of any study is relevant to its abstract whatever included there should be elaborated later in the protocol.

Abstract writing: First explain scientific background/ rationale for the investigation being reported. Elaborate abstract with: problem and rational. Provides background information about issue you are dealing with, state a research question. Address the topic in first sentence and introduce topic by means of an example/ /studies to illustrate points. Outline your general argument/specific points.

How to write an abstract is the puzzle. Investigators should write an abstract based on the researched question and the searched information. The structured abstract needed to be for not more than A4 one page. This constitutes the most meticulous step in research, successful protocol, study implementation and publication in a decent journal. Structured abstract informs about the most important stations for the train to keep on the track. If any station is not well-defined and well-addressed, then the whole train misses that stop and ramifies far away. A simple structured abstract can help any clinical researcher, who wants to go systematic and wants smooth flow of his study and publication, is given in Table A, (1).

Writing an abstract consists primarily of answering the questions, "Why did you start?" What is research problem why important? "What did you do and why?" "What did you find?" and "What does it mean?", (2).

The components of the structured abstract are not easy to build and these questions should be all addressed: What is your aim(s), objective(s)? Is it a prospective or a retrospective study design and why? Will the study setting be an outpatient, discharged, inpatient or the public? What will constitute the population from where you draw your sample? How will you to calculate the sample size? Which technique will be used? In the event that your study is about a new method the last 2 questions might be changed to: "What are advantages (of method)? and "How well does it work? The title/structure of the abstract/study must be dynamic/informative not descriptive. One should use succinct writing, perfect grammar language and short sentences, (3-7).

Your study introduction and background: The background is the clinical research's backbone, where you can support your clinical research hypothesis. You have to explore your clinical research idea and what is known in the past, what is the current status and how your research will contribute to the future, $(8,9)$.

In an original research, the manuscript introduction should focus specifically on the hypothesis/aim of study. Furthermore, it must not be an exhaustive review but rather recommended to range from maximum 2 to 5 paragraphs. There should be paragraphs of main substations as international experience, continental, regional, country or local community. Typically, the introduction part must be designed in the format of a cone, as also suggested by Annesley, (Figure 1), (1). Initiating what is known information, the unknown information-gap, hypothesis/ purpose statement (problem) and plan-approach-solution.

\begin{tabular}{|c|l|}
\hline \multicolumn{2}{|c|}{ Table A Ten Questions to ask When Critically Appraise Research Article. } \\
$\begin{array}{c}\text { Serial } \\
\text { Number }\end{array}$ & \multicolumn{1}{|c|}{ Questions to critically appraise research article } \\
\hline 1 & Is the study question relevant? \\
\hline 2 & Does the study add anything new? \\
\hline 3 & What type of research question is being asked? \\
\hline 4 & Was the study design appropriate for the research question? \\
\hline 5 & $\begin{array}{l}\text { Did the study methods address the most important potential } \\
\text { sources of bias? }\end{array}$ \\
\hline 6 & Was the study performed according to the original protocol? \\
\hline 7 & Does the study test a stated hypothesis? \\
\hline 8 & Were the statistical analyses performed correctly? \\
\hline 9 & Do the data justify the conclusions? \\
\hline 10 & Are there any conflicts of interest? \\
\hline
\end{tabular}


Table B Strengths and Weaknesses of Quantitative Evaluation Designs.

\section{Strengths}

- Study findings can be generalized to the population about which information is required.

- Samples of individuals, communities, or organizations can be selected to ensure that the results will be representative of the population studied.

- Structural factors that determine how inequalities (such as gender inequalities) are produced can be analyzed.

- QUANT estimates can be obtained of the magnitude and distribution of impacts.

- QUANT estimates can be obtained of the costs and benefits of interventions.

- Clear documentation can be provided regarding the content and application of the survey instruments so that other researchers can assess the validity of the findings.

- Standardized approaches permit the study to be replicated in different areas or over time with the production of comparable findings.

- It is possible to control for the effects of extraneous variables that might result in misleading interpretations of causality (although this can be challenging in natural settings of evaluations).

\section{Weaknesses}

- Many kinds of information are difficult to obtain through structured data collection instruments, particularly on sensitive topics such as domestic violence or income.

- Many groups such as sex workers, drug users, illegal immigrants, squatters and ethnic minorities are always difficult to reach, but problems are often greater for QUANT data collection methods.

- Self-reported information obtained from questionnaires may be inaccurate or incomplete.

- There is often no information on contextual factors to help interpret the results or to explain variations in behavior between households with similar economic and demographic characteristics.

- The administration of a structured questionnaire creates an unnatural situation that may alienate respondents.

- Studies are expensive and time consuming and even the preliminary results are usually not available for a long period of time.

- Research methods are inflexible because the instruments cannot be modified once the study begins.

- Reduction of data to numbers results in lost information.

- Correlations produced (e.g., between costs/benefits, gender, and access to services or benefits) may mask or ignore underlying causes or realities.

- Untested variables may account for program impacts.

- Errors in the hypotheses tested may yield misimpressions of program quality or influential factors.

- Errors in the selection of procedures for determining statistical significance can result in erroneous findings regarding impact.

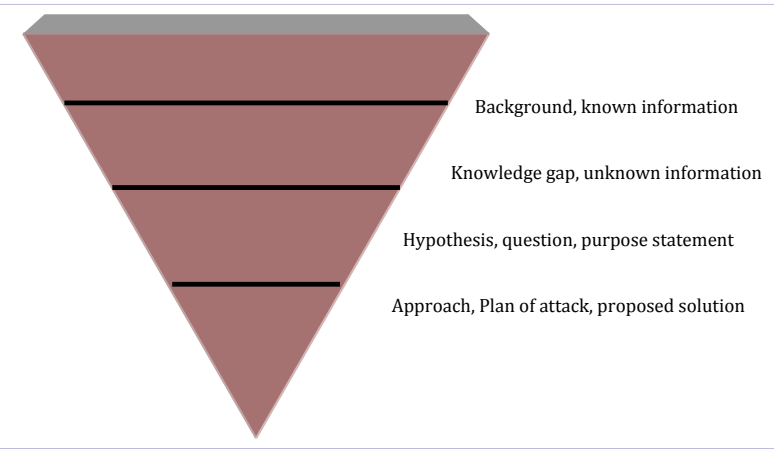

Figure 1: Cone format for writing "Introduction" by Annesley.

What is known information?: The introduction is your gate to readers, where you explore the international experiences on the research topic (compare, define, describe, evaluate, examine, explain, explore, investigate, etc...) in the first paragraph. It should focus on "what is known" about your hypothesis. "Introduction" should include a quick summary of all other studies on your selected topic and what other international researchers have determined by their studies. You can start comparing the results of with the most recent randomized controlled trials if there are any, then; you can move to meta-analysis, systemic reviews, longitudinal cohort studies, observational studies and then case series. Next, you have to do is to focus should be the regional literatures, what the researchers from neighbor countries have investigated and what the peers in your country have found Then, you can move to compare and contrast these with the international studies. At the end, you should focus on the gaps; the limitations of the previous studies that are have particular importance while comparing their results to your present study.
Highlighting of what is missing and what would be needed can make the comparison more fruitful.

Hypothesis/burning question/aim: This section should be clear in order to enlighten your own vision and your message to the research community. You may wish to inform about the rational of your current study, and what it will add to the body of evidence. Aims are 'what you want to achieve' it is a general intention, broader vision, is to close gap in knowledge (i.e. answer research question). We achieve aim by providing a contribution to knowledge. The objective (s) is "how you intend to go about this" "How do objectives fit in? Describe steps taken to achieve aim? Describe sub aims you need to achieve to achieve overall aim?

\section{Materials and Methods}

\section{Research approach}

A clinical researcher may wish to inform the other investigators on the clinical implications of the current study; its clinical significance and the most important contribution to fill in the gap of the field or discipline you is writing about, state differences and anticipate new practices.

\section{Tips for writing an "Introduction"}

- Keep paragraphs short, clear, concise, non-technical and should be focus onto general readers.

- First write "What is Known?"; later "What is Unknown?" and later focus on your "Hypothesis".

- Emphasize how your study fills in the gaps of the previous literature (i.e. the unknown). 
- Explicitly state your research question/aim/hypothesis to be clear.

- Do not defense or disclose the research question.

- Summarize what has been done on your research subject before and give all the detailed descriptions, speculations, and criticisms of those studies.

\section{Ethics approval}

That is your transparency with the world, did you apply for ethical approval or you did not. If you did, where if you do not you need to do. Ethical approval is another station where we rest and take a breath to continue the journey. The ethics committee, whatever you name or call it and where ever it denoted is the body that grants you the authentication to perform your research. It is your stamped study and your well reviewed and registered trademark (as you may intend to register your study with known entities as clinicaltrial.gov).

It is a chance for your study to get weighed, assessed, peerreviewed and corrected with integrity and coherence to follow standard research. Lucky you, if your ethics committee has an Object Identifier (OI) as Pub med and others. The OI is another way to denote originality of work intended for publication and international view.

How to fill your ethics committee forms and play your symphony is another technical task with skills emphasis. You need to practice this and ask your peers to judge your work before you go out well dressed. Once an approval is granted from the ethics committee, you should be proud of your work and stick to the rules and to your study protocol. Minor changes are possible provided that you refer back to the ethics committee and carry on. Now your project is blooming and not dare to enter the practical part of implementing your conceptual and hypothesis.

Needless to say you need to present to the ethics committee the patient informed consent form which details your study to the patient (add an information sheet) and seek his/her acceptance to participate in the study with the patient right to withdraw at any point without giving any reasons. There are ethical, good clinical practice and regulatory requirements that should be viewed from international authorities such as the Helsinki declaration and others.

\section{Results for methodology}

When, where, and how was the study done? What materials were used or who was included in the study groups (patients, etc.)? The components of the methodology are enormous and vary considerably according to the type of study. The following are typical subheadings: the study design, study setting, population, sample size, patient selection, inclusion and exclusion criteria, data collection / instrument / procedure, outcome measures and statistical tests.

The study design is crucial and determinant to the success of the study. The investigator's need to select the suitable study design that delineate the research question problem, data captured and solution to the problem. The followings are the types of study design you may chose from: observational studies (case study, case control, case series, cohort/cross sectional), randomized control trials (open label, single-blind, double = blind), quasi experiment (non RCT), data mining, meta-analysis, networking meta-analysis, and systematic reviews.

\section{Describe the study setting}

Where is the study locations (private versus government), outpatient versus inpatient, community/clinic versus hospital, which clinical area/ward, is it a multi setting and more details about each selected location in term of bed-capacity, personnel, specializing and so falls.

Specify dates for commencing the study and draw a schematic description with dates. The period of recruitment of patients, period of exposure and follow-up dates should be clearly outlined.

\section{The Data variable}

The description of methods/procedures for obtaining data should be thought of very carefully. The reasons for choosing the methods/procedures should be clarified and discussed with reasoning. Describe the types of data (qualitative/quantitative) and sources from where these data will be obtained, retrieved and reported. The data variables are the foundation of the research and where you arrange the red bricks of the great palace for building your study. Before you even launch your study, you should prepare a data-set. These data-sets should always contain the population demographics, socio-demographics and anthropometrics details of study population. Report the pertinent patient medical/ medication histories, diagnosis, morbidity/ mortality and relevant risk factors. The entire laboratory data and relevant tests specific to the research in questions should be included in the data sheet, preferable in "Excel" sheets or directly into a predefined statistical data variable sheet e.g. SPSS. In a complex patient profile, you may add as much as you can, with particular attention to general versus specific laboratory variables. You should leave space for causal variables that may appear during your research and odds findings which are not anticipated at initial study conceptual framework.

The data collection and storage should follow the applicable data protection regulations. There are many proper data management tools like the quintiles (https://eportal.quintiles. com) which provide web-based a data capturing tool that store the filled clinical data and ensure organized meaningful clinical inferences.

\section{Outcome measure and statistical tests}

A brilliant clinical researcher will define each correlation within the variables of the study. The integration between the components of the abstract is the solution of a difficult puzzle. The relationship between the data variables, outcome measure and the statistical tests is one of the most critical points in clinical research, particularly in clinical/medical, comparative, clinical toxicology, efficacy and safety trials. If a researcher finds a relationship between any two variables, he should 
explain the mechanism by which these two are correlated. The correlations found between the variables, which are analyzed by the appropriate statistical process, will yield the outcome measure. Therefore, defining the correlations consist the most crucial step in clinical outcome research. Description of methods and procedures of data analysis should be performed in liaison with statistical expert and given the time and the needed build of skills.

The development of clear outcome measure definitions and objective outcome measure definitions is of paramount importance. The outcome measure (s) should correspond to the nature of the hypothesized treatment effect. The outcome measure should address research questions of interest. The validation of outcome measure (s) or use of standardized patient reported outcome measure instruments validated for population of interest that contribute to internal validity of studies is too indicative for reliable findings and generalization of results. Attention should be given to the collection of outcome data in an equivalent manner across treatment comparison groups. Use appropriate analytic methods suitable to outcome. Apply sensitivity analysis to address varying definitions of primary/ secondary/major/ intermediate/composite study outcome measure (s) to draw robust and reliable inferences.

The outcome measure (s) as (clinical and humanistic) is the end-results of particular healthcare practices, policies and interventions. They provide evidence about benefits, risks, and outcome of treatments/practice change/audit and guidelines implementation. The outcome types may be observation, patient and clinician reported. Few examples as improved clinical status, improved health, improved quality of care/health/life, lowered morbidity or mortality, improvement of abnormal states/lab values, and emphasis on effectiveness (effect of an intervention as applied to broad populations in real practice). The properties of outcome (subjective/objectives) as integral part of an investigator's evaluation selection of appropriate measures include: reliability, Validity and variability, (10-13).

\section{Combining Results}

\section{Results}

What answer was found to the research question; what did the study find? Was the tested hypothesis true? You should put your statistical outputs on organized predefined titled tables, no matter how many initially, but later you can deduce to the minimum number of tables. You may start your tables by the demographics; past medical/medication/ morbidities etc. Then, you can narrow down to your main findings. Each table stands as your discussion area. Therefore, tables must follow an uphill fashion and upward direction to the final conclusions. The tip of the iceberg is your main findings. Detail the main findings and provides a summary explanation of results and indicate whether you accept or reject the hypotheses.

The guidelines on how to write reports are available and should be consulted such as (CONSORT (http://www.consortstatement.org/) for clinical trials/STROBE (http://www.strobestatement.org/) for epidemiological studies.

\section{Discussions}

What might the answer imply and why does it matter? How does it fit in with what other researchers have found? What are the perspectives for future research? The discussion is your study background and introduction where you can explore the findings and compare to international studies. You can start your discussion by your main findings and then, you can discuss the less important results. Develop a logical argument about what your results mean. The reported results should provide evidence to illustrate and support your argument. Results discussions need to identify potential errors, and what might invalidate your findings. Also you may add how you may improve the research design. Occasionally, you need to discuss your methodology and procedures, and pros and cons. The aligned differences between the study findings and other studies should be reasoned and explained with clear statements.

\section{Clinical Research Strengths and Weaknesses}

In order to assess its strengths and weaknesses, the clinical research must comply with all outlined ethical standards, regulations and good clinical practice as appropriate.

Discussing the strengths and weaknesses of a clinical study, is more or less a critical appraisal of one's own research before it is evaluated by reviewers and weighed in relevancy to successful publication (Table B).

Strengths: Strength of a clinical research can be summarized as:

- The usefulness and validity of the presented clinical research findings: It is the evaluation of the appropriateness of the study design for a defined research question. A careful assessment of the key methodological features can justify the outcomes of your results.

- Selection bias,

- Confounding factors,

- The suitability of the statistical techniques used and their subsequent interpretations,

- Bias-type of error,

- Potential conflicts of interest,

- The relevance of the clinical research to one's own clinical practice.

Weaknesses: This should be written in away to reflect the true obstacles arisen during the conceptual frame and study design. You should report: whatever drawbacks been encountered during the study, even with data collection and interpretations. The study weaknesses should not be viewed as negative points rather than inevitable events and circumstances that have not been expected from the time when study hypothesis is built to the time when findings are obtained and conclusions are drawn. If well- stated, study weaknesses will provide readers more opportunities to design better future studies that overcome such pitfalls of the presented study. Furthermore, it will be a 
learning opportunity for the authors to improve their research skills and revert back with more rigorous research.

Clinical Significance or Study Implications: The presented clinical research must comply with the clinical real scenarios, real practice and the necessity to change/intervene and implement the clinical study output. When the clinical significance is matching with the undertaken research and its yield, a more practicable conclusion could be drawn. The study implications should answer whether results are applicable to practice; what is the magnitude of risk and harm, if there are any.

\section{Summary}

Commencing the research on structured abstract manner will lead to successful study implementation and publishable manuscript. Therefore, it is imperative to perform extensive and up to date review by using authenticated search engines such as, PubMed, Cochrane and systematic reviews to explore your research question/topic/area. More elaborated existing guidelines are available for those who wish to develop clinical trial protocols (SPIRIT (http://www.spirit-statement. org/about-spirit/the-people-of-spirit/).

\section{Conclusion}

Conclusion is the pearl of the research. It is your message that tells the core of what is presented and what will be explored in the near future. You can conclude with the most important findings from the performed work, without jumping to new facts or mitigating the real results. You should report both the main and minor findings. You can recommend changes and interventions that can facilitate the undertaken clinical research findings. You can keep the future prospects of the presented subject to give the reader the chance to carry from here to forward; however you should not to address new questions and leave unanswered phrases. You should further detail how your findings can be applicable to the day-to-day clinical practice and make generalization to the population from which the data are pertaining. You can explain how your present work can be the research-gate for other investigators to emulate and cite the study.

\section{References}

1. Annesley TM. "It was a cold and rainy night": Set the scene with a good introduction. Clin Chem. 2010; 56(5):708-13. doi: 10.1373/ clinchem.2010.143628.

2. Pierson DJ. How to write an abstract that will be accepted for presentation at a national meeting. Respir Care. 2004, 49(10): 120612.

3. Alexandrov AV, Hennerici MG. Writing good abstracts. Cerebrovasc Dis. 2007; 23(4):256-9.

4. Baillie J. On writing: write the abstract, and a manuscript will emerge from it! Endoscopy. 2004; 36(7):648-50.

5. Christensen NB, Kume $H$, Autorino R. How to write titles and abstracts for readers. Int J Urol. 2009; 16(1):2-3. doi: 10.1111/j.14422042.2008.02228.x

6. Cummings P, Rivara FP, Koepsell TD. Writing informative abstracts for journal articles. Arch Pediatr Adolesc Med. 2004; 158(11):1086-8.

7. Durbin CG, Jr. Effective use of tables and figures in abstracts, presentations, and papers. Respir Care. 2004; 49(10):1233-7.

8. Sengupta S, Shukla D, Ramulu P, Natarajan S, Biswas J. Publish or perish: The art of scientific writing. Indian J Ophthalmol. 2014; 62(11):1089-1093. doi: 10.4103/0301-4738.146760.

9. Korieth K. The Center Watch Monthly. 2008; 15(12):8-13.

10. Streiner DL, Norman GR. Health Measurement Scales: a Practical Guide to their Development and Use. 4th ed. Oxford University Press; 2008.

11.U.S. Department of Health and Human Services, Food and Drug Administration. Clinical Outcome Assessment Qualification Program, 2012.

12. Kip KE, Hollabaugh K, Marroquin OC, Williams DO. The problem with composite end points in cardiovascular studies. The story of major adverse cardiac events and percutaneous coronary intervention. J Am Coll Cardiol. 2008; 51(7):701-7. doi: 10.1016/j.jacc.2007.10.034.

13. U.S. Department of Health and Human Services, Food and Drug Administration. Guidance for Industry Patient Reported Outcome Measures: Use in Medical Product Development to Support Labeling Claims. Dec, 2009. 